\title{
Methods of data mining for modelling of low-pressure heat treatment
}

\author{
E. Wołowiec-Korecka \\ Institute of Material Science and Engineering, Lodz University of Technology, \\ ul. Stefanowskiego 1/15, 90-924 Łódź, Poland \\ Corresponding e-mail address: emilia.wolowiec@p.lodz.pl
}

\section{ABSTRACT}

Purpose: This paper addresses the methods of the modelling of thermal and thermochemical processes used in computer-aided design, optimization and control of processes of thermal and thermochemical treatment in terms of obtaining real-time results of the calculations, which allows for observation of how an item changes during its treatment to respond immediately and to determine the parameters of a corrective process should any irregularities be detected. The main goal of the literature review was to develop a methodology for the design of functional and effective low-pressure processes of thermal and thermochemical treatments using effective calculation methods.

Design/methodology/approach: A detailed analysis was conducted regarding the modelling methods with low-pressure carburizing and low-pressure nitriding.

Findings: It was found the following criteria of methods selection of heat treatment modelling should be applied: data quality, data quantity, implementation speed, expected relationship complexity, economic and rational factors.

Practical implications: Because of its non-equilibrium nature and transient states in the course of the processes computational support is particularly required in low-pressure thermochemical treatments. The primary goal of the simulation is to predict the course of the process and the final properties of the product, what ensures the repeatability of the process results.

Originality/value: It was presented a synthetic presentation of modelling methods, in particular methods of artificial intelligence; it was also analysed the possibilities and risks associated with methods.

Keywords: Heat treatment, Low-pressure carburizing, Low-pressure nitriding, Data mining

\section{Reference to this paper should be given in the following way:}

E. Wołowiec-Korecka, Methods of data mining for modelling of low-pressure heat treatment, Journal of Achievements in Materials and Manufacturing Engineering 85/1 (2017) 31-40.

\section{ANALYSIS AND MODELLING}




\section{Introduction}

The basis of an effective thermal and thermochemical treatment is to capture the relationships between the treatment processes, the microstructure and the postprocess properties of a material. The ability to identify and control the parameters affecting the intensity of the processes is the key to achieving the desired combination of material features required for a specific application. Understanding these relationships is the starting point for further actions, such as optimization of a sample or the initial state of a material, e.g. composition of alloys, in terms of effectiveness, duration and cost of treatment.

A number of studies have been conducted in recent years on the phenomena that occur during low-pressure thermochemical processes, including nitriding and carburizing. Considering the above, the interest in this subject matter is understandable as its topicality remains high. It is also an important issue for practitioners to achieve a high level of conformity of the real items under treatment with the technological assumptions. Unfortunately, process optimization is one of the most difficult issues in material engineering research. Luckily, conducting research by a trial-and-error method is gradually being abandoned and IT methods, computeraided modelling of processes and correlations in thermal and thermochemical treatment are becoming increasingly popular. This is caused by users' increasing expectations and the pressing need to control costs, which necessitates changes in production processes, including process design and control. Moreover, the wide availability of computers and their growing computational capabilities is not without importance. This has stimulated interest in process modeling and applications for thermal and thermochemical treatment. This applies to the processes themselves and to the post-process properties of items under treatment [1-5]. Therefore, this paper discusses the computation methods applied in computer-aided thermal and thermochemical treatment processes, with the aim of achieving better conformity of the actual post-treatment properties with the designed ones, which will contribute to the improved repeatability of the processes.

Detailed investigations concerning low-pressure carburizing and low-pressure nitriding were carried out. The main purpose of the literature review was to better understand the cause and effect relationship in these processes and to develop the methodology for the design of functional and effective low-pressure thermal and thermochemical treatment processes using effective calculation methods. The article contains a synthetic presentation of modelling methods, in particular methods of artificial intelligence; it also analyses the opportunities and risks associated with these methods.

As expectations regarding the quality and precision of product manufacture continue to grow, further advances in materials engineering are closely associated with the use of computer-aided computation methods. As has been said before, a traditional method of achieving the optimum material properties by trial and error is time-consuming and costly, which is an argument for seeking alternative ways of designing thermal and thermochemical treatment processes.

Computer simulations and modelling simplifies the design of engineering materials and prediction of the properties of these materials with a significant reduction of time and costs; hence there are numerous reports on this subject [6-12].

Using data mining (DM) methods is an interesting trend, which has been used in modelling and simulations for several years. For gas nitriding, there are models which employ fuzzy logic, heuristic models, artificial neural networks, evolution algorithms and other similar methods $[13,14]$. Using neural networks or genetic algorithms is not common in thermal and thermochemical treatment due to the need for gathering vast training databases, but owing to the capabilities which are inaccessible to the conventional mathematical methods, the number of studies in which such methods are employed has been steadily growing [15-29].

Because of its non-equilibrium nature and transient states during processes computational support is particularly required in low-pressure thermo-chemical treatments. In this case, the main purpose of the simulation is to predict the course of the process and the final properties of the product. It ensures repeatability of the process results [30,31].

What is important about the practical usability of technological process-aiding tools is that they can be used in real time. It is desirable to be able to control a process online and to respond immediately if any irregularities are detected, preferably through automatic design and initiation of a corrective process. This necessitates using quick algorithms and computation methods and it is the primary objective of this paper to systematize, examine them and assess their usability in thermochemical treatments.

\section{Data mining methods}

The literature on the subject provides grounds for the classification of modelling techniques into two distinct groups: physical and statistical, each with its own 
advantages and areas of application. Both groups of methods are constantly being improved and applied to model different processes and correlations, both in producing primary material (metallurgy) and in its further treatment by material engineering methods.

Physical methods are usually based on fundamental laws and theories which describe the physical nature of the process. Therefore, it is the basic assumption in this kind of modelling to understand the phenomenon perfectly and to be able to describe it using a physical model and mathematical equations, as in modelling thermodynamic phenomena based on Gibbs' equations and in Thermo-Calc and MTDATA software, which has been successfully applied in thermodynamic calculations in different systems [32-35]. However, when the knowledge of a phenomenon is incomplete, then the traditional methods of data analysis and an analytical approach are insufficient. This applies to situations when the analysis of a large amount of data is needed and a number of parameters need to be taken into account which can have a significant effect on the process features or on the item which is being processed. It also happens - as is the case in non-equilibrium processes - that the relationships between the process parameters and the features of the item under treatment are unknown and it is risky to make assumptions regarding the type of relationship they have to each other (e.g. that it is linear). Such cases necessitate a flexible approach and use of "intelligent" learning methods. In consequence, methods are commonly known as data mining methods or, more broadly, KDD (Knowledge Discovery in Databases) methods, are increasingly popular; these involve the use of data mining methods as well as database-related techniques.

There are a number of definitions of data mining, but the majority of them confirm that it is a certain way of handling, processing and analysing data. To quote Berry and Linoff, one could say that it is "a process of studying and analysing large amounts of data by automatic and semi-automatic methods aimed at discovering significant patterns and rules" [36,37]. Furthermore, Migut and Demski mention four key features that distinguish data mining from traditional statistical data analysis [38]. These are:

1. Ability to analyse large bodies of data.

2. Orientation towards practical results and applications rather than on developing and testing theories.

3. Applying methods which are capable of reproducing virtually any relationship between variables.

4. Using existing data first.

Model quality assessment is based on a test sample, usually taken from an experimental database.
A data mining analysis consists of three parts. The first one is the extrapolation which begins with data preparation. Data is cleared (e.g. of redundant, contradictory, dubious data) and transformed into a usable form. Subsequently, the choice of a case sub-set and a preliminary choice of variables (features of the phenomenon, which are regarded as significant) are made. The aim of these actions is to reduce the number of variables under analysis to a rational level, i.e. such that enables an effective analysis. Further actions depend on a specific issue. Different methods are used, from a simple choice of variables by linear regression to examination by graphic and statistical methods, in order to finally determine the most important features as well as the complexity and the general nature of the phenomenon [39].

A model construction, assessment of correctness and usability of results is the proper data analysis stage. It is here that various methods are considered which can reproduce the nature of the phenomenon under consideration and the best of them is chosen. The quality of prediction, i.e. whether the value of the variable being modelled is correct, and the stability of results for different samples is the criterion of the assessment. Although at first, the choice of the best model seems to be a relatively easy task, in practice it may turn out to be a complicated process. Techniques based on comparative model evaluations, which involve the use of individual methods for the same data sets and choosing the best of them or developing a complex model, are frequently applied. Techniques of model evaluations and joining are regarded as a key part of predictive data mining. They include model aggregation (voting and averaging the responses), amplification (adaptive resampling and combining), model contamination and meta-learning. The model found to be the best at this proper stage of data analysis passes on to the final stage where it is applied to new data. The aim of the application of this model is to obtain predicted values or classification [36-38].

As has been said earlier, the choice of a model depends on the task set before it. According to Berry [36], two principal groups of tasks include predictive issues and discovering new knowledge, with predictive issues including classification, regression and time series, and discovering new knowledge is understood to denote: detecting deviations, segmentation, cluster analysis, analysis of associations and sequences, summary listings, visualization and text mining. The studies conducted for this work concerned primarily the predictive issues, with the main emphasis placed on regression and classification, whereas an extensive description of the whole subject matter can be found in papers by Weiss, Ratner, Hassti, Braha and others [36-47]. 
Predictive issues involve situations when there is a need for developing a model which can be used to predict a value of a feature (dependent variable) from a set of other features (independent variables, such as process parameters). A model is trained on an example set of objects (cases) for which both independent and dependent variables are known. Predictive issues include classification and regression (which are important for the subject matter of the article).

The following are applied to solve regression problems: linear regression, regression trees, linear models and generalized linear models, MAR Splines, neural networks, boosted trees, the $\mathrm{k}$ nearest neighbours
(k-NN) method, which can be useful in supplying missing data), SVM (support vector method). The following methods are applied to solve classification problems: neural networks, classification trees, discriminative analysis, logistic regression, generalized linear models, general additive models, boosted classification trees, k-NN method, naive Bayes classifier, SVM, MAR Splines $[36,37,39,40,48]$. The effectiveness of a given method (or a group of methods) depends on the objectives of the analysis, the structure, and quality of data; therefore, Migut and Demski analysed literature on the subject and suggested [38] that its assessment should be aspectrelated, as in Table 1 .

Table 1.

A review of selected methods of modelling [38]

\section{Methods of modelling:}

1. NN, 2. SVM, 3. Trees, 4. MARS, 5. K-NN, 6. Linear models (GLM, GRM, GDA), 7. Boosted trees, 8 . Naive Bayes method

\begin{tabular}{|c|c|c|c|c|c|c|c|c|}
\hline & 1 & 2 & 3 & 4 & 5 & 6 & 7 & 8 \\
\hline $\begin{array}{l}\text { Handling data of various } \\
\text { types naturally }\end{array}$ & - & - & + & + & - & - & + & + \\
\hline Missing data & - & - & + & + & + & - & $+/-$ & - \\
\hline Robustness to outliers & - & - & + & - & + & - & + & - \\
\hline $\begin{array}{l}\text { Scalability } \\
\text { (N observations) }\end{array}$ & - & - & + & + & - & + & - & $+/-$ \\
\hline $\begin{array}{l}\text { Scalability } \\
\text { (N variables) }\end{array}$ & - & - & + & $+/-$ & $+/-$ & + & $+/-$ & $+/-$ \\
\hline $\begin{array}{l}\text { Robustness to } \\
\text { insignificant variables }\end{array}$ & - & - & + & + & - & $+/-$ & - & - \\
\hline Interpretability & - & - & + & $+/-$ & - & $+/-$ & - & + \\
\hline Predictive power & + & + & - & $+/-$ & + & - & + & - \\
\hline
\end{tabular}

NN - Neural Networks; SVM - Support Vector Machine; MARS - Multivariate Adaptive Regression Splines method; k-NN - k Nearest Neighbours algorithm; GLM - Generalized Linear Models; GRM - General Regression Models; GDA - Generative Learning Algorithms

\section{Calculations}

The following section contains a presentation and analysis of two models of thermochemical treatment processes which employ the methodology described above and which were chosen following data analysis, also described in the previous section.

\subsection{Modelling single-phase diffusion in low-pressure carburizing}

An atmosphere based on hydrocarbons as carbon carriers is characterized by very high carbon potential. The disequilibrium nature of vacuum carburizing imposes a division of the vacuum carburizing process into a series of phases, in this into in cycles of repeatable stages of boost and diffusion (Fig. 1) in order to avoid the creation of by-products and to reach a required carbon profile $[49,50]$. The model of vacuum carburizing, its development and the development of technology based on it has been described in $[51,52]$.

The model enables the designing processes of vacuum carburizing as well as the analysis and optimization of the process without having to conduct time-consuming and costly testing. Depending on the user's needs, it predicts the outcome of the process in the form of a carbon profile or designs the process by itself in terms of the desired case properties. 


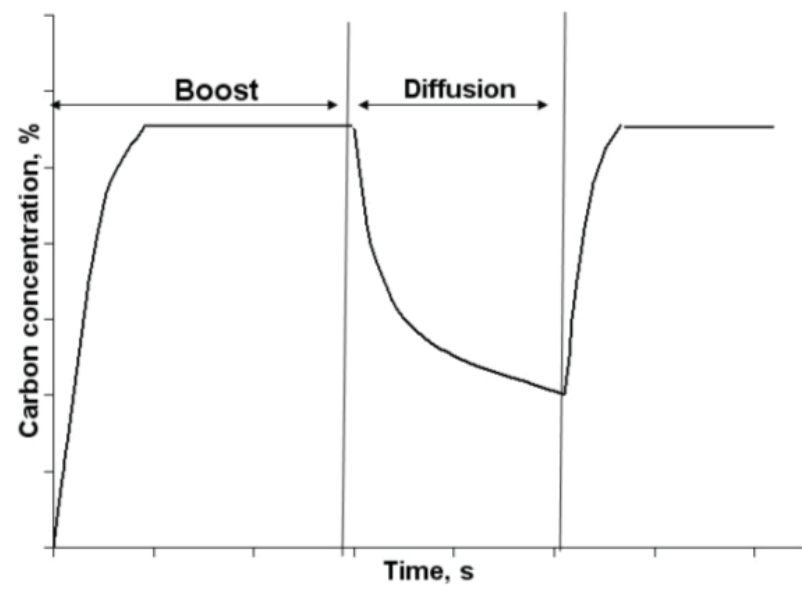

Fig. 1. Carbon concentration in austenite during the development of boost and diffusion stages

In its calculations, the model takes into account a range of parameters: the steel grade, the shape, and geometry of the items, the charge surface area, the carbon concentration on the surface, the case depth criterion, the carburizing temperature as well as the time sequence for the boost and diffusion segments. A further phase of calculations takes into account the cooling phase before quenching, the type, and pressure of the cooling gas and the size of the furnace; however, these features are beyond the scope of this article [53-57]. As a result, the model achieves a high accuracy of predictions for actual processes.

The mathematical-physical model of low-pressure carburizing process under consideration is based on six principles defined by Kołodziejczyk, Kula and others in $[58,59]$ :

1. Carburizing atmosphere in the saturation stage is delivered to the sample surface in a continuous manner.

2. The composition of the carburizing atmosphere remains constant throughout the process.

3. Carbon atoms are released as a result of a catalytic interaction between the atmosphere and the surface being carburized.

4. The coefficient of carbon diffusion in austenite is a function of the temperature, carbon concentration and the presence of alloy additives.

5. Carbon is transported to the material according to Fick's equations (diffusion's laws).

6. A semi-infinite area has to be taken into consideration in calculations.

The model of low-pressure carburizing which fulfils these assumptions, with subsequent quenching in high pressured gas, is a hybrid model, in which:
- basic phenomena of low- and medium-carbon steels carburizing was described by an analytical mathematical-physical model;

- the coefficient of carbon transfer and the carbon diffusion coefficient was determined by analysis of variance and multiple regression;

- phenomena which could not be described by mathematical equations, such as the creation and dissolution of carbide formations, were analysed by means of data mining methods (neural networks);

- phenomena of the formation and dissolution of carbon deposits were described by a heuristic model;

- the model of cooling and equations of unsteady heat flow were based on CFX numerical methods;

- the area and weight of the charge were estimated by means of conventional mathematical apparatus;

- the "cooling down rate - carbon content - material hardness" relationship has been tabularized, what speeded up the calculations and make to obtain results in real time available;

- the material data regarding the steels were collected in a database to eliminate redundant processes and accelerate the model operation;

- a separate model of vacuum carburizing for atypical materials, which is beyond the assumptions of the base model, has been developed.

\subsection{Modelling multi-phase diffusion in low-pressure nitriding}

The model of low-pressure nitriding, described in $[55,60,61]$ is intended for the designing, simulating and optimizing processes of multi-segment low-pressure nitriding of items made of tool steels. Founded on methods of artificial intelligence, the model enables for the implementation of the multi-stage technology of lowpressure nitriding - FineLPN as a technological option to be carried out in multi-purpose vacuum furnaces. Its aim is to choose the optimum parameters of a technological process for a specific steel grade in order to modify its surface layer appropriately at as low a cost as possible.

The construction of the system was preceded by the accumulation of a vast training database, concerning the course of low-pressure nitriding processes, needed to examine and describe the kinetics of the processes, in particular to develop a mathematical and neural model of growth of a uniform nitrided case on tool steels, with a desired hardness profile and structure - the optimum one in terms of the conditions in which the tools are to be used. 
The operation of the program is based on a modular structure. The computational modules operate independently, exchanging the required information, whereas the computational core of the system is a module which implements methods of artificial intelligence, especially the operation of artificial neural networks. The module implements a physical model and its functional solution for multi-segment low-pressure nitriding in a "boost-diffusion" type process.

The program can operate in one of two variants. It can simulate the properties of the top layer following a userdesigned process or it can suggest its own process parameters as long as the user defines the expected strength properties of an item after treatment.

The latter option is more interesting from the operational point of view. In the first step, the system acquires input data regarding the item's post-process properties. After the data is converted to the universal system of units, the system initiates the artificial intelligence module. Data of an item (steel grade, expected hardness, expected case depth) is then fed to the neural network inputs. The algorithm is looking for a process which can produce the desired properties within the shortest possible time. In the next step, the process of lowpressure nitriding is simulated with the parameters suggested by the network and the full post-process characteristics of the material are presented (hardness, total case depth, the range of occurrence of the white layer and the dark layer, possibly a lattice of precipitations along grain boundaries).

Low-pressure nitriding has been studied by Kula, Wołowiec and others and their findings have been reported in [56,59-62]. The most important parameters which affect the kinetics of growth of the nitrided layer and its phase composition in the gas nitriding process include: the nitrogen potential, temperature, the composition and flow rate of the nitriding atmosphere, the composition of the material being nitrided and the preparation of the surface which is to be nitrided. In lowpressure nitriding, the nitrogen potential no longer matters, but another parameter appears - the process segmentation because the process of low-pressure nitriding is divided into alternate stages of material saturation and holding at a nitriding temperature without nitriding gas. The assumptions for the phenomena which occur during the nitriding process, and which arise from the phenomenological model of the process, have been presented in work [56].

1. The build-up of the nitrided layer takes place during alternate stages of saturation with nitrogen and holding;
2. The nitriding atmosphere during the saturation stage is supplied to the sample surface in a continuous manner, at a constant flow rate;

3. The composition and pressure of the nitriding atmosphere remain constant during the saturation stages;

4. The nitriding gas is not supplied during the holding phase, nor does it remain in the nitriding space;

5. Nitrogen atoms are acquired as a result of the desorption of the product of ammonia dissociation;

6. Nitrogen is transported into the material by diffusion in accordance with Fick's law;

7. The coefficient of diffusion in the material depends on the temperature, concentration of nitrogen and presence of alloy additions.

The relationships between the parameters of the lowpressure nitriding process and the features of the nitrided layer are complicated because of the non-equilibrium nature of low-pressure nitriding. This has been pointed out by Dobrodziej [53], who claimed that "solving Fick's equations of diffusion, which describe the transport of nitrogen atoms necessary to form the surface layer, by analytical or numerical methods is a considerable challenge, for example due to the non-linear relationship between the diffusion coefficient and the rate of diffusion".

The system of equations which describe the relationships can be solved only with a simplified approach to the phenomena which accompany the nitriding process is followed, which results in unacceptable discrepancies between the calculations and the observations. The problem can - as yet - be solved only by an optimization procedure, which takes into account several variable parameters, the assumption being that the others are constant. Considering the above, the analytical approach to solving the problem has been given up in this model and the data analysis by the data mining method was applied. The model of lowpressure nitriding was based totally on the neural network method; moreover:

- evaluation of the steel types and the specific nature of the nitriding process was made by applying neural networks to model the classification problems,

- diffusion kinetics in multi-phase systems and the relationships of phase formation: diffusion, $\gamma^{\prime}$ and $\varepsilon$ phases, were reproduced with neural networks for modelling regression problems.

\subsection{Seeking the optimum process parameters}

Reversal of the direction of links between the input parameters of the carburizing and the nitriding processes and their outcome values aimed at finding the best process 
parameters is one of the optimization issues. Evolutionary algorithms, in particular, genetic ones, are the most precise tools that can be used to solve these kinds of problems $[9,25,27,62-64]$. However, this solution is not acceptable as it requires the model to work on a real-time basis. Therefore, there are two possible models: a mathematicalheuristic one and one that uses neural networks. A heuristic model is usually developed by a trial-and-error method. It is a combination of physical equations and engineering simplifications. Its characteristic feature is that it "defends itself" by the conformity of its output data with results of real-life experiments; however, there is no mathematical proof that it is the best existing solution to the problem. Such a solution has been adopted in models described in [65-67]. An algorithm based on a neural network as an alternative method of conducting a process has been described in [54]. Calculations are made very quickly in the first case; in the other, there is a time-consuming network training stage, but this is a one-off action.

\section{Discussion}

The data contained in Table 1 has numerous implications. For example, using neural networks may require data of an appropriate quality (credible, free of noise and free of outliers) and it may involve complicated calculations, but it allows one to reproduce even very complicated relationships, although it does not allow for a model interpretation (it is a "black box" type model). Moreover, a model based on the use of the neural network is poorly extrapolable; therefore, it is recommended that training patterns should cover the whole domain of the problem under consideration. The SVM has similar properties. On the other side of the properties, there are regression trees, which do not require special data preparation measures, but their predictive capabilities are small. The k-NN method and the boosted trees method show a considerable similarity of features. Linear methods and the Bayes naive method are also similar, with the linear models being more resistant to insignificant variables and the Bayes naive method being able to better handle various types of data.

The literature on the subject $[40,48]$ suggests that seeking one's own solutions should be started with simple models and methods, which can be made increasingly complex as needed. The author of this paper has also applied various methods in her numerous studies, not discriminating against the simplest ones as long as they proved sufficiently effective. This is manifest in the lowpressure carburizing model, which was described by analytic equations in properly explored aspects and supplemented by more complex methods, such as data mining methods (neural networks, heuristics), databases and CFX type numerical methods.

As has been mentioned earlier, the relationship between the parameters of the nitriding process and the outcome features of the material in a low-pressure nitriding process are complex; therefore, with reference to the characteristics of artificial intelligence methods described in Table 1 and the selection criteria presented above, a method of artificial neural networks were used to reproduce them. The method has a certain drawback in that the model cannot be interpreted, as it is a "black box" type model; however, the inconvenience has been accepted because of the network advantages and a general model of the process has been proposed, which has been described in [55].

\section{Conclusions}

A literature review regarding the methods of designing and manufacturing modern materials has shown that computer-aided modelling of phenomena and features of materials is becoming common. Computer-aided reproduction of the processes and phenomena helps to forecast properties of materials with a considerable reduction of the financial outlays and the time needed for experiments and implementation.

This study presents a comprehensive review of various methods of modelling and their applications in thermal and thermochemical treatment. It is beyond doubt that contemporary progress of material engineering is closely associated with the application and development of methods of mathematical modelling, numerical methods and artificial intelligence.

The methods described above have been used to develop and present two effective models and applications which employ them to aid processes of thermal and thermochemical processes. They operate in real time, which makes it possible to apply them successfully in the industry. The correctness and effectiveness of the solutions have been verified on the basis of real-life experimental databases.

\section{References}

[1] L.A. Dobrzański, J. Trzaska, Application of neural networks for the prediction of continuous cooling transformation diagrams, Computational Materials 
Science 30/3-4 (2004) 251-259, doi: 10.1016/j. commatsci.2004.02.011.

[2] P. Cavaliere, G. Zavarise, M. Perillo, Modeling of the carburizing and nitriding processes, Computational Materials Science 46 (2009) 26-35, doi: 10.1016/j. commatsci.2009.01.024.

[3] I.B. Ozdemir, N. Lippmann, Modeling and simulation of surface reactions and reactive flow of a nitriding process, Surface and Coatings Technology 219 (2013) 151-162.

[4] O. Karabelchtchikova, R.D. Sisson, Calculation of gas carburizing kinetics from carbon concentration profiles based on direct flux integration, Defect and Diffusion Forum 266 (2007) 171-180.

[5] O. Karabelchtchikova, I.V. Rivero, S.M. Hsiang, Modeling of residual stress distribution in D2 steel via grinding dynamics using a second-order damping system, Journal of Materials Processing Technology 198/1-3 (2008) 313-322, doi: 10.1016/j.jmatprotec. 2007.07.006.

[6] P. Jacquet, D.R. Rousse, G. Bernard, M. Lambertin, A novel technique to motor carburizing process, Materials Chemistry and Physics 77 (2002) 542-551.

[7] H. Larsson, J. Agren, Gas nitriding of high vanadium steels - experiments and simulations, Metallurgical and Materials Transactions A 35/9 (2004) 2799-2802.

[8] T. Malinova, S. Malinov, N. Pantev, Simulation of microhardness profiles for nitrocarburized surface layers by artificial neural network, Surface and Coatings Technology 135 (2001) 258-267.

[9] S. Fang, M. Wang, Y. Wang, W. Qi, Evolutionary artificial neural network approach for predicting properties of $\mathrm{Cu}-15 \mathrm{Ni}-8 \mathrm{Sn}-0.4 \mathrm{Si}$ alloy, Transactions of Nonferrous Metals Society of China 18/5 (2008) 1223-1228.

[10] Z. Gawroński, J. Sawicki, Technological surface layer selection for small module pitches of gear wheels working under cyclic contact loads, Materials Science Forum 513 (2006) 69-74, doi: 10.4028/www. scientific.net/MSF.513.69.

[11] S. Lipa, J. Sawicki, E. Wołowiec, K. Dybowski, P. Kula, Method of determining the strain hardening of carburized elements in Ansys environment, Solid State Phenomena 240 (2016) 74-80.

[12] Z. Gawroński, J. Sawicki, Toothed wheel optimization by means of the finite element analysis, Mechanics and Mechanical Engineering - International Journal 4/2 (2000) 183-189.

[13] J. Dobrodziej, A. Mazurkiewicz, J. Wojutyński, J. Michalski, J. Ratajski, Model of controlling gas nitriding processes in the precise creation of surface layers with programmed properties, Journal of the Japan Society for Heat Treatment 49 (2009) 769-773.

[14] J. Ratajski， R. Olik, T. Suszko，J. Dobrodziej, J. Michalski, Design, Control and in Situ Visualization of Gas Nitriding Processes, Sensors 10/1 (2010) 218240, doi: 10.3390/s100100218.

[15] S. Malinov, W. Sha, Software products for modelling and simulation in materials science, Computational Materials Science 28 (2003) 179-198, doi: 10.1016/ S0927-0256(03)00106-X.

[16] M. Aliofkhazraei, A.S. Rouhaghdam, Neural networks prediction of different frequencies effects on corrosion resistance obtained from pulsed nanocrystalline plasma electrolytic carburizing, Materials Letters 62 (2008) 2192-2195. doi:10.1016/j.matlet.2007.11.052.

[17] L.A. Dobrzański, W. Sitek, Comparision of hardenability calculation methods of the heat-treatable constructional steels, Journal of Materials Processing Technology 64 (1997) 117-126.

[18] L.A. Dobrzański, W. Sitek, Application of a neural network in modelling of hardenability of constructional steels, Journal of Materials Processing Technology 78 (1998) 59-66.

[19] K. Genel, I. Ozbek, A. Kurt, C. Bindal, Boriding response of AISI W1 steel and use of artificial neural network for prediction of borided layer properties, Surface and Coatings Technology 160 (2002) 38-43.

[20] K. Genel, Use of artificial neural network for prediction of ion nitrided case depth in $\mathrm{Fe}-\mathrm{Cr}$ alloys, Materials and Design 24/3 (2003) 203-207, doi: 10.1016/S0261-3069(03)00002-5.

[21] S. Malinov, W. Sha, Application of artificial neural networks for modelling correlations in titanium alloys, Materials Science and Engineering A 365 (2004) 202211, doi: 10.1016/j.msea.2003.09.029.

[22] S. Malinov, W. Sha, Z. Guo, Application of artificial neural network for prediction of time-temperaturetransformation diagrams in titanium alloys, Materials Science and Engineering A 283 (2000) 1-10.

[23] N.S. Reddy, J. Krishnaiah, S. Hong, J. Lee, Modeling medium carbon steels by using artificial neural networks, Materials Science and Engineering A 508 (2009) 93-105, doi: 10.1016/j.msea.2008.12.022.

[24] W. Sha, K.L. Edwards, The use of artificial neural networks in materials science based research, Materials and Design 28 (2007) 1747-1752, doi: 10.1016/j.matdes.2007.02.009.

[25] W. Sitek, L.A. Dobrzański, Application of genetic methods in materials' design, Journal of Materials Processing Technology 164-165 (2005) 1607-1611, doi: 10.1016/j.jmatprotec.2005.01.005. 
[26] W. Sitek, L.A. Dobrzański, J. Zacłona, The modelling of high-speed steels' properties using neural networks, Journal of Materials Processing Technology 157-158 (2004) 245-249, doi: 10.1016/j.jmatprotec.2004.09.037.

[27] R.G. Song, Q.Z. Zhang, Heat treatment technique optimization for 7175 aluminium alloy by an artificial network and a genetic algorithm, Journal of Materials Processing Technology 117 (2001) 84-88.

[28] L. Xu, J. Xing, S. Wei, Y. Zhang, R. Long, Optimization of heat treatment technique of highvanadium high-speed steel based on back-propagation neural networks, Materials and Design 28 (2007) 1425-1432, doi: 10.1016/j.matdes.2006.03.022.

[29] A. Zhecheva, S. Malinov, W. Sha, Simulation of microhardness profiles of titanium alloys after surface nitriding using artificial neural network, Surface and Coatings Technology 200 (2005) 2332-2342, doi: 10.1016/j.surfcoat.2004.10.018.

[30] M. Korecki, P. Kula, J. Olejnik, New capabilities in HPGQ vacuum furnaces, Industrial Heating 3 (2011), http://www.industrialheating.com/articles/89834-newcapabilities-in-hpgq-vacuum-furnaces? $\mathrm{v}=$ preview (accessed June 2, 2017).

[31] H. Klumper-Westkamp, H. Zoch, Sensor Application in Heat Treatment Processes for Enhancement of the Process Capability, Proceedings of the $3^{\text {rd }}$ International Conference Heat Treating 2011, Qual. Heat Treat., IFHTSE, Wels, 2011, 98-105.

[32] K. Evanson, G. Krauss, D. Medlin, M.J. Patel, Bencin Fatigue Behaviour of Vacuum Carburized AISI 8620 Steel, Proceedings of the $2^{\text {nd }}$ International Conference Carburizing Nitriding Atmosphares, Cleveland, 1995, 61-69.

[33] CFX-4.2 Solver, AEA Technology, CFDS Department, Harwell, 1997.

[34] W.E. Dowling, T. Pattok, B.L. Ferguson, et al., Development of a carburizing and quenching simulation tool: Program Overview, HTM Journal of Heat Treatment and Materials 1 (1997) 1-6.

[35] M. Dall'Oro, S. Villa, D. Valtolina, F.M. Montevecchi, The Carburizing of Steel with Hydrocarbon Gas in Low Relative Pressure, Proceedings of the $11^{\text {th }}$ International Conference Heat Treatment and Surface Engineering, Florence, 1998, 487-492.

[36] M.J. Berry, G. Linoff, Data mining techniques: for marketing, sales and customer support, John Willey \& Sons, Hoboken, 1997.

[37] M.J. Berry, G. Linoff, Mastering data mining, John Willey \& Sons, Hoboken, 2000.

[38] G. Migut, T. Demski, Technical information, 2012.
[39] Statistica. Data Miner, StatSoft, Cracow, 2002.

[40] S.M. Weiss, N. Indurkhya, Predictive data mining. A practical guide, Morgan Kaufmann Publisher, Burlington, 1998.

[41] D. Braha, Data mining for design and manufacturing. Methods and applications, Kluwer Academic Publisher, Heidelberg, 2001.

[42] P. McCullagh, J.A. Nelder, Generalized linear model, Chapman \& Hall, London, 1999.

[43] A. Agresti, An introduction to categorical data analysis, John Willey \& Sons, Hoboken, 1996.

[44] B. Ratner, Statistical modeling and analysis for database marketing, Chapman \& Hall, London, 2003.

[45] J. Han, M. Kamber, Data mining: Concepts and techniques, Academic Press, Waltham, 2001.

[46] J. Friedman, Multivariate adaptive regression splines, The Annals of Statistics 19/1 (1991) 1-67.

[47] D. Pyle, Data preparation for data mining, Academic Press, Waltham, 1999.

[48] T. Hastie, R. Tibshirani, J. Friedman, The elements of statistical learning, Springer-Verlag, Heidelberg, 2002.

[49] P. Kula, J. Olejnik, P. Heilman, Hydrocarbon gas mixture for the under pressure carburizing of steel, EU $1558780,2007$.

[50] P. Kula, J. Olejnik, P. Heilman, Hydrocarbon gas mixture for the under-pressure carburizing of steel, 7,513,958, 2009.

[51] P. Kula, R. Pietrasik, K. Dybowski, Vacuum carburizing - process optimization, Journal of Materials Processing Technology 164-165 (2005) 876-881.

[52] P. Kula, K. Dybowski, E. Wołowiec, R. Pietrasik, Boost-diffusion vacuum carburizing - process optimisation, Vacuum 99 (2014) 175-179.

[53] J. Dobrodziej, J. Wojutynski, K. Matecki, A. Gospodarczyk, J. Michalski, J. Tacikowski, P. Wach, J. Ratajski, R. Olik, The possibility of use of computer applications to design, simulation and verification of the processes of regulated gas nitriding, Surface Engineering 14/2 (2009) 34-45 (in Polish).

[54] E. Wolowiec, The Application of Artificial Intelligence Methods in Development and Technical Realization of Surface Engineering Processes, PhD Thesis, Lodz University of Technology, 2009.

[55] E. Wolowiec, P. Kula, B. Januszewicz, M. Korecki, Mathematical modelling the low-pressure nitriding process, Applied Mechanics and Materials 421 (2013) 377-383.

[56] E. Wolowiec, Computer design of heat treatment processes, Lodz University of Technology, Lodz, 2013. 
[57] M. Korecki, P. Kula, E. Wolowiec, M. Bazel, M. Sut, Low pressure carburizing and nitriding of fuel injection nozzles, Heat Treatment Reprots 3 (2014) 59-62.

[58] L. Kolodziejczyk, Mathematical modeling of the process of vacuum carburizing, $\mathrm{PhD}$ Thesis, Lodz University of Technology, 2003.

[59] E. Wolowiec, P. Kula, Ł. Kolodziejczyk, K. Dybowski, M. Korecki, Mathematical modelling of the vacuum carburizing process, Thermal Processing for Gear Solutions 3-4 (2014) 34-40.

[60] P. Kula, R. Pietrasik, E. Wolowiec, B. Januszewicz, A. Rzepkowski, Low-Pressure Nitriding according to the FineLPN Technology in Multi-purpose Vacuum Furnaces, Advanced Materials Research 586 (2012) 230-234.

[61] P. Kula, E. Wolowiec, R. Pietrasik, K. Dybowski, B. Januszewicz, Non-steady state approach to the vacuum nitriding for tools, Vacuum 88 (2013) 1-7, doi: 10.1016/j.vacuum.2012.08.001.
[62] R.G. Song, Q.Z. Zhang, Heat treatment optimization for 7175 aluminum alloy by genetic algorithm, Materials Science and Engineering C 17/1-2 (2001) 133-137, doi: 10.1016/S0928-4931(01)00321-6.

[63] D. Goldberg, Genetic algorithms, Addison-Wesley Reading, Boston, 1989.

[64] T. Velsker, M. Eerme, J. Majak, Artificial neural networks and evolutionary algorithms in engineering design, Journal of Achievements in Materials and Manufacturing Engineering 44/1 (2011) 88-95.

[65] M. van Wie, Heuristics, science, and engineering, Mechanical Engineering - CIME 134 (2012) 8.

[66] P. Kopecek, Selected heuristic methods used in industrial engineering, Procedia Engineering 69 (2014) 622-629, doi: 10.1016/j.proeng.2014.03.035.

[67] E.S. Mistakidis, G.E. Stavroulakis, Nonconvex optimization in mechanics: algorithms, heuristics and engineering applications by the F.E.M., Kluwer Academic Publisher, Heidelberg, 1998. 